\title{
Adesão às Listas de Medicamentos Essenciais por Médicos Brasileiros em Atuação no Sistema Único de Saúde
}

\author{
PALAVRAS-CHAVE \\ - Medicamentos Essenciais; \\ - Educação Médica; \\ - Médicos de Atenção \\ Primária; \\ - Médicos Hospitalares; \\ - Assistência Farmacêutica; \\ - Sistema Único de Saúde; \\ - Brasil.
}

\section{Adherence to the Essential Medicines Lists by Brazilian Physicians in the Unified Health System}

Rachel Magarinos-Torres ${ }^{I}$ Ângela Esher ${ }^{I I}$

Rosângela Caetano ${ }^{I I I}$

Vera Lúcia Edais Pepe ${ }^{I I}$

Claudia Garcia Serpa Osorio-de-Castro ${ }^{I I}$

\begin{abstract}
RESUMO
Objetivo: Investigou-se a adesão de médicos brasileiros em atuação no SUS a listas de medicamentos essenciais (LME), buscando conhecer o papel das LME na prática prescritiva e identificar a aceitação e barreiras para sua utilização no Brasil. Métodos: O estudo, de âmbito nacional, entrevistou médicos da Atenção Primária e da hospitalar de 30 unidades públicas de saúde de municípios com e sem LME definida. Na análise dos dados foram utilizadas técnicas da pesquisa qualitativa em saúde. As categorias finais de análise foram: (i) contato com diferentes LME; (ii) utilização das LME na prática clínica; (iii) percepção do conceito de medicamentos essenciais. Resultados: Foram ouvidos 58 médicos, sendo 11 do Nordeste e do Centro-Oeste e 12 do Sudeste, Norte e Sul. Apenas 17 dos 58 médicos entrevistados informaram contato anterior com uma LME, a maior parte referindo-se à lista municipal. Quando perguntados se utilizavam a Rename em sua prática clínica, todos os entrevistados responderam que não. Dentre os motivos citados, estão (i) a indisponibilidade dos medicamentos (da lista) no momento requerido; (ii) a falta de orientação necessária para o uso; (iii) a impressão de que a composição da lista é inadequada à demanda clínica. Conclusões: Os resultados das falas expõem desconhecimento e baixa adesão a LME. Ainda que tenham tido algum contato prévio com uma LME, esta não é valorizada como fonte de informações para a prescrição baseada em evidências.
\end{abstract}

\begin{abstract}
Objective: To investigate the adherence to the essential medicines lists (EML) by Brazilian physicians practicing in the public health system and the role of the EML in prescribing practices, identifying barriers to their use in Brazil. Methods: A nationwide study interviewed physicians from diverse public healthcare settings in 30 facilities, in municipalities with different statuses in regard to the existence of EMLs. Research data was analyzed through content analysis and perception analysis techniques. The final analytical categories were (i) physician's contact with different EMLs, (ii) use of EMLs in clinical practice and (iii) physician's perceptions regarding the essential medicines concept. Results: A total of 58 physicians were interviewed, from all five Brazilian regions: eleven from the Northeast, eleven from the Mid-West, and twelve each from the Southeast, the North and the South. Seventeen of the 58 physicians reported previous contact with an EML, most of which had occurred with municipal lists. All physicians informed that they did not use the Brazilian Essential Medicines List (RENAME) in their clinical practices. Among the main reasons for this were: (i) unavailability of listed medicines at required moment, (ii) the lack of necessary information and training for adequate use of EML, and (iii) the perception that the composition of the EML is inadequate for clinical demands. Conclusions: Results from content analysis exposed low awareness regarding EMLs and the essential medicines concept, in addition to a low level of adherence to EMLs. Even though some physicians reported having previous contact with an EML, this tool is not valued as an evidence-based information source for writing prescriptions.
\end{abstract}

REVISTA BRASILEIRA DE EDUCAÇÃO MÉDICA
${ }^{I}$ Faculdade de Farmácia, Universidade Federal Fluminense, Niterói, RJ, Brasil.

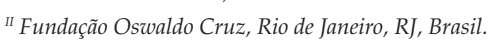

III Universidade do Estado do Rio de Janeiro, Rio de Janeiro, RJ, Brasil. 


\section{INTRODUÇÃO}

A adoção do conceito de medicamentos essenciais é internacionalmente reconhecida como uma medida capaz de racionalizar o uso de medicamentos com resultados benéficos para a clínica e para a gestão. O conceito preconiza que o melhor uso é aquele que considera as necessidades prioritárias da população e cuja operacionalização passa pela elaboração de uma relação qualificada, denominada genericamente lista de medicamentos essenciais (LME) ${ }^{1}$.

A escolha dos medicamentos que irão compor a relação de medicamentos essenciais, um processo chamado de seleção, deve considerar as prioridades sanitárias, a prevalência das doenças, a eficácia e a segurança dos medicamentos, e as avaliações de custo-efetividade. Por definição, a seleção é um processo dinâmico de análise das necessidades em saúde e dos ganhos efetivos das novas opções terapêuticas ${ }^{1}$. O processo é feito com base em dados epidemiológicos e em evidências científicas por um comitê multidisciplinar que legitima a LME. Esse comitê é denominado Comissão de Farmácia e Terapêutica (CFT)².

Durante cerca de 15 anos, o Brasil investiu intensamente na elaboração e divulgação de sua Relação Nacional de Medicamentos Essenciais (Rename), que passou a ser revista com regularidade a partir de $2000^{3}$. Cabe destacar que a primeira Rename foi publicada em 1964, antes mesmo da recomendação da OMS. A preparação e divulgação de uma lista de medicamentos essenciais de modo condizente com o conceito é sempre um esforço coletivo. A edição da Rename publicada em $2010^{4}$, por exemplo, foi desenvolvida durante aproximadamente 18 meses e demandou 17 reuniões presenciais de dois dias de duração com especialistas de diferentes instituições educacionais e de saúde no País, sob a coordenação do Ministério da Saúde (MS), e produziu, além da lista em si, 55 pareceres para subsidiar gestores e prescritores.

Ao longo do tempo, a difusão das diversas edições da Rename incluiu publicação on-line e impressa com distribuição para estados e municípios brasileiros. Foram também investidos recursos na elaboração e distribuição de duas edições do Formulário Terapêutico Nacional ${ }^{5}$, contendo informações técnicas qualificadas de cada um dos 372 fármacos presentes nesta Rename.

Permanece, no entanto, o problema da adesão de prescritores às listas de medicamentos essenciais em diferentes regiões brasileiras ${ }^{6,78}$. Somam-se a isto os novos desafios impostos à gestão pelo crescente aumento da demanda por medicamentos, aliados à imposição legal de provisão, mesmo em situações de constrangimento orçamentário e gestão incipiente 9 .

A literatura discute o fenômeno das dificuldades de adesão a protocolos, diretrizes e listas por parte de prescritores, apontando que um dos eixos deste fenômeno se relaciona ao conhecimento e à percepção sobre as $\mathrm{LME}^{10}$. A não adesão dos médicos às listas de medicamentos essenciais é um risco para o paciente e para o sistema de saúde, seja pela pressão que exerce na gestão da Assistência Farmacêutica, com impactos na logística de medicamentos e nos orçamentos da saúde, seja pela exposição, muitas vezes desnecessária, dos pacientes a medicamentos com fracas evidências científicas para a indicação proposta e com risco de ocorrência de efeitos adversos. O fenômeno da judicialização da saúde caracteriza-se, em grande parte, pela prescrição não aderente às $\mathrm{LME}^{11,12}$.

A pesquisa intitulada "Fala Essencial" (2009-2010) investigou a percepção de gestores e de médicos do Sistema Único de Saúde (SUS) em relação ao conceito de medicamentos essenciais e o papel das LME na organização da Assistência Farmacêutica no Brasil e na prática prescritiva ${ }^{13}$. Este texto apresenta e discute os resultados referentes à adesão dos médicos brasileiros em atuação no SUS aos medicamentos essenciais, buscando conhecer o papel das LME na prática prescritiva e identificar a aceitação e as barreiras para sua utilização no Brasil.

\section{MÉTODO}

O estudo foi desenvolvido com base no referencial teórico-metodológico da pesquisa qualitativa em saúde, tendo por unidade de análise prescritores médicos com atividade profissional no Sistema Único de Saúde.

A escolha dos informantes visou abranger a diversidade brasileira quanto aos modelos de atenção à saúde em diferentes cenários de gestão da Assistência Farmacêutica. A presença de LME foi adotada como um marcador dos processos realizados e definiu o "contexto" Estado/município. De início, foi sorteado aleatoriamente um Estado dentre os quatro Estados brasileiros que apresentavam, no momento da pesquisa, lista de medicamentos essenciais estadual (Rememe) publicada ${ }^{13}$. Para as demais regiões brasileiras, foi sorteado um Estado, nenhum dos quais com Rememe divulgada.

De cada um desses Estados, foi incluída a capital e sorteados mais dois municípios; segundo dados disponibilizados pelo Instituto Brasileiro de Geografia e Estatística (IBGE) em 2010. Para cada município, foram sorteadas duas unidades públicas de saúde, uma voltada ao atendimento ambulatorial e outra ao hospitalar, perfazendo seis unidades por Estado. A identificação das unidades foi realizada com auxílio do Cadastro Nacional de Estabelecimentos de Saúde consultado em 2010. A inclusão das unidades de saúde respeitou a condição pública da unidade, a existência de atendimento direto a pacientes e a presença de pelo menos dois médicos vinculados à unidade.

Foi realizado contato telefônico e por correio eletrônico com cada uma das unidades envolvidas. Mediante aceite pre- 
liminar dos gestores da unidade, foram convidados às entrevistas dois médicos por unidade de saúde, o que totalizaria 60 médicos, sendo dois por município e dez por região. A fase de contato telefônico com os gestores durou aproximadamente seis meses, e as entrevistas, quatro meses, sendo ambas executadas em 2010.

Cinco farmacêuticos treinados atuaram como entrevistadores. Os roteiros incluíram características dos participantes e questões relacionadas com sua formação e atuação profissional. A coleta buscou conhecer a posição dos entrevistados sobre o conceito de medicamentos essenciais e sobre as LME para sua prática prescritiva.

As entrevistas foram gravadas, transcritas e analisadas com auxílio da Técnica de Análise de Conteúdo ${ }^{14}$ e do Método de Interpretação dos Sentidos ${ }^{15}$. A leitura flutuante do material permitiu elaborar uma matriz de análise com as ideias trazidas pelos sujeitos por categoria e temática. A leitura horizontal desta matriz de análise forneceu o conjunto de ideias apresentadas e, com isto, o estabelecimento de relação entre os depoimentos.

As categorias finais de análise foram: (i) contato com LME; (ii) utilização das LME na prática clínica; (iii) percepção do conceito de medicamentos essenciais. O julgamento quanto a "contato com uma LME" considerou o conjunto de afirmações realizadas pelo entrevistado que considerasse: (a) quanto ao conceito de LME: o entendimento da lista como o produto de um processo de seleção de medicamentos essenciais em qualquer esfera administrativa - local, municipal, estadual ou federal -, não sendo considerada menção à lista de referência para aquisição de medicamentos ou referência à relação dos medicamentos disponíveis naquele momento no estoque da unidade; (b) quanto ao termo "contato com a LME": desde o manuseio e a utilização da lista propriamente dita, até uma breve aproximação por referência de terceiros (sic).

A pesquisa foi desenvolvida de acordo com as diretrizes e normas para pesquisas com seres humanos contidas na Resolução 196/1996 do Conselho Nacional de Saúde. O projeto foi aprovado pelo Comitê de Ética em Pesquisa da Escola Nacional de Saúde Pública Sergio Arouca em 25 de março de 2010 CAAE: 0218.0.031.000-9. Para ampliar o caráter sigiloso, os informantes foram codificados segundo sexo e categoria, sendo alocados nomes fictícios a cada um, iniciados pela letra " $\mathrm{L}$ ".

\section{RESULTADOS E DISCUSSÃO}

\section{Perfil dos Entrevistados}

Foram ouvidos 58 médicos brasileiros no Sistema Único de Saúde, sendo: 11 do Nordeste, 11 do Centro-Oeste, 12 do Sudeste, 12 do Norte e 12 do Sul. Esta composição aconteceu em decor- rência de divergências entre a informação presente no CNES e o número real de médicos em duas unidades de saúde, resultando em um prescritor a menos no Nordeste e no Centro-Oeste.

Compuseram o grupo 20 mulheres e 38 homens, com idade entre 29 e 77 anos, sendo 37 egressos (63,7\%) de universidades públicas. Foram declaradas 11 diferentes especialidades médicas (Resolução CFM n $\left.{ }^{\circ} 1.666 / 2003\right) ; 33$ prescritores se autorreferiram como "clínico geral" e 26 (44,8\%) informaram atuar tanto na Atenção Primária como na hospitalar.

Dos 58 médicos ouvidos, 22 trabalhavam nas capitais $(37,9 \%)$ e 36 no interior $(62,1 \%)$. O tempo de atuação no SUS variou de menos de um ano ( $5 \%$ dos prescritores) a mais de 30 anos $(10 \%)$, sendo o desvio padrão de 9,8 e a mediana ao redor de 15 anos, na capital, e de 11 anos, no interior.

Dado o tempo de trabalho no SUS, haveria possibilidade de conhecimento e contato com diferentes versões da LME nacional pela maior parte dos entrevistados. As LME incluem medicamentos usados em baixa, média e alta complexidade e privilegiam situações prioritárias de saúde que se concentram na baixa e média complexidade ${ }^{1}$. Adicionalmente, destaca-se que, sendo a configuração de atuação dos prescritores predominante para baixa e média complexidade, é esperada proximidade deste grupo de prescritores com LME.

\section{Contato com Listas de Medicamentos Essenciais}

Apenas 17 (29,3\%) dos 58 médicos entrevistados informaram contato prévio com uma LME, mesmo sendo tão ampla a definição adotada. Destes, 6 mencionaram a Rename, 8 (13,7\%) a LME municipal (Remume) e 3 a LME do hospital onde atuavam. Nenhum prescritor fez menção à Rememe, embora tenham sido visitados dois Estados com lista.

Os resultados apontam que a aproximação entre o prescritor e a lista nacional (Rename) parece ter pouca relação com a existência de LME estadual ou municipal. Os dois extremos de cenário vislumbrado - presença de LME no município e no Estado, e ausência de LME nas duas esferas - tiveram prescritores que conheciam a Rename. Ficou patente o completo grau de desconhecimento dos prescritores sobre a Relação Estadual de Medicamentos Essenciais.

Dada a posição que as LME possuem na estruturação da Assistência Farmacêutica (AF) no Brasil e a contribuição da AF para a consolidação do SUS $9,16,17$, estes resultados causam preocupação. A seleção de medicamentos, com os padrões de qualidade enunciados pela OMS é reconhecida, na Política Nacional de Medicamentos (PNM), como uma diretriz prioritária na ampliação do acesso e na promoção do uso racional de medicamentos. Todas as ações enunciadas pela PNM para a AF ancoram-se na seleção de medicamentos essenciais. A seleção 
insere-se no Ciclo da Assistência Farmacêutica como um momento de organização das demais ações, incluindo a prescrição, dado que é responsável por eleger os medicamentos que deverão estar disponíveis e que, assim, serão utilizados ${ }^{1,16,18}$. A maior parte desses prescritores mencionou a relação municipal, muito embora a proporção tenha sido menor que a esperada, dado o perfil de atuação dos prescritores. A adesão dos prescritores às listas pressupõe um programa de medicamentos essenciais inserido em uma política de AF eficiente ${ }^{19} \mathrm{e}$ implantada no município. A divulgação da relação da esfera municipal parece estar sendo mais efetiva, muito embora todos os prescritores entrevistados trabalhassem em unidades de saúde com serviço de farmácia na própria unidade, o que favorece, por si só, o contato com as listas municipais ${ }^{8}$.

O contato dos prescritores com a Rename ocorreu no espaço profissional por diferentes formas e motivações. Em alguns casos, o conhecimento aconteceu em decorrência de restrições de uso a determinados medicamentos:

"Eu conheço assim, quase de ouvir falar [...] Através de um problema que deu aqui. [...] O farmacêutico embasou o parecer dele para não incluir essa medicação. Eu vi o livro [...] falando de efeitos colaterais, de reações adversas e tal. E foi assim que eu conheci. Mas fora isso, eu não conheço muita coisa de Rename". (Lineu)

Essa fala expressa certo estranhamento em relação à lista. A lista é usada por outro profissional; ele não a conhece bem, não faz parte da sua prática, e sim do espaço de atuação do farmacêutico. Por outro lado, aponta que o farmacêutico usou a Rename, indicando que ele a conhece e a utiliza. Não foi estabelecido, entretanto, um diálogo construtivo entre estes profissionais capaz de estimular a desejada prática multidisciplinar baseada em evidências ${ }^{20,21}$. Esta situação corre o risco de se tornar mais aguda com a recente introdução, pelo Governo Federal, de uma Rename que reúne todos os medicamentos presentes em listas de financiamento público ${ }^{22}$, estando, assim, mais caracterizada como lista de compra do sistema do que como lista de medicamentos essenciais - portanto, distanciada do conceito de integralidade e das necessidades em saúde, o que não contribui para a formação de prescritores no SUS.

Em outras situações, a aproximação das LME foi mediada pelas coordenações municipais, com o aparente intuito de informar a disponibilidade de itens:

"Foi em uma apresentação da Assistência Farmacêutica da Secretaria Municipal de Saúde [...]. Mostraram para vocêtomar ciência das medicações e justificar os medicamentos com disponibilidade da Rename". (Leonidas)
Percebe-se que, para alguns profissionais, o contato com a Rename é pontual e burocrático, está na ordem da sigla e não na ordem da utilidade prática ou da possibilidade de formação para o uso racional:

"Já li alguma coisa, Relação Nacional de Medicamentos, não é isso? Mas isso na época em que foi feita a lista, não é? Que a gente teve mais contato com essas siglas. Sei do que se trata, não é? Basicamente". (Luis Pedro)

Parece ter havido pouco investimento na formação continuada sobre medicamentos essenciais, uma exigência para que a relação seja efetiva e adequadamente apropriada pelos profissionais em prática cotidiana. Em países onde a implantação das listas de medicamentos essenciais é exitosa, com ganhos importantes para a terapêutica e para o sistema, há considerável empenho em realizar contatos pessoais com prescritores e em capacitá-los ${ }^{19}$.

\section{Utilização das LME na Prática Clínica}

Quando perguntados se utilizavam a Rename em sua prática clínica, todos os médicos entrevistados responderam que não. Dentre os motivos apresentados, surgiram vários, dentre eles: (i) indisponibilidade dos medicamentos (da lista) no momento requerido; (ii) falta de orientação necessária ao uso; (iii) impressão de que a composição da lista é inadequada à demanda clínica:

\section{"[...] fiquei com a sensação de que era muito limitada [...] que você não tem os medicamentos no estoque". (Lorival) \\ "Ninguém nos orientou a usar [...]. Não sei a importância dela. [...]" (Lineu) \\ “Eram poucos medicamentos. Medicamentos às vezes que já estavam consagrados nos estudos ou na prática nossa fica- vam de fora, não é?" (Luis Pedro)}

Esses motivos mostram que, na percepção do prescritor, a "orientação" quanto ao uso da lista deve ser "fornecida" e não buscada pelo próprio médico, como parte da necessária atualização profissional. Não houve propensão a se adequar à prescrição de medicamentos da lista, mas, sim, de forma recorrente nas falas, o entendimento da lista como limitada e inadequada à "sua" prática. Muito do que os prescritores trazem se deve, possivelmente, a desconhecimento ou a hábitos arraigados de prescrição ${ }^{6}$. Como apenas 29,3\% relataram contato prévio com a lista em si, é possível supor que um percentual mais baixo teria realmente conhecimento mais aprofundado do seu conteúdo. 
Ainda, a LME foi vista como uma ameaça à autonomia médica:

"Nenhuma norma administrativa, [...] onde quer que seja, pode se sobrepor à autonomia médica". (Lineu)

Na tipologia mintzberguiana ${ }^{21}$, são exigidos altos níveis de qualificação dos operadores nas organizações profissionais, e, devido à complexidade e, por vezes, imprevisibilidade do trabalho, é necessário que tenham alto grau de autonomia. Por outro lado, esta autonomia, fundamental à qualidade dos serviços, deve ser submetida ao controle, de forma a integrar as ações desenvolvidas no contexto da organização e do sistema, e não pode se contrapor ao fluxo desse sistema. Neste sentido, a autonomia médica exercida de modo a desconsiderar os preceitos da essencialidade e da evidência científica, nas quais se baseia o conceito de medicamentos essenciais, não se apresenta como a melhor prática, seja para o sistema, seja para o paciente individual.

Resultados semelhantes quanto à percepção de interferência na autonomia foram encontrados por estudos internacionais que se dedicaram a analisar a aceitação da $\mathrm{LME}^{24,25}$. Uchoa e Camargo ${ }^{18}$ mostraram que clínicos cumpririam rotinas e protocolos desde que estes não restringissem sua autonomia técnica. Na região de Estocolmo, os prescritores aderem a uma lista de cerca de 200 medicamentos, e seu grau de adesão é de $98 \%$, sem que vejam nisso ameaça a sua autonomia ${ }^{19}$.

A não utilização da Rename é também associada a maior ou melhor atualização do médico ou à sua especialização. É conferido valor adicional à prescrição de medicamentos que não estão na Rename:

\footnotetext{
"[...] dentro do próprio SUS, quando a gente encaminha para um especialista, geralmente eles não usam o que está na Rename [...] Eu entendo por que eles prescrevem muitas coisas que não tem (na lista), porque muitas vezes eles estão atualizados com estudos que mostram algum benefício maior para uma determinada medicação que ainda não está na Rename". (Lucio Mauro - clínico geral)
}

Este resultado também mostra desconhecimento com relação ao escopo da Rename, que, entre 2000 e 2010, albergou baixa, média e alta complexidade, com pelo menos a primeira e a segunda opção terapêutica para as prioridades em saúde no País. Por outro lado, a fala acima mostra que a concepção de "qualidade" pode estar associada a novidade. Esse entendimento, contudo, não reconhece que muitas das "novidades" não passam de pequenas modificações em produtos já existentes no mercado, dado que o constante lançamento de "novos" produtos é uma das estratégias da indústria farmacêutica para a manutenção de suas fatias de mercado. Nem sempre estes novos produtos, contudo, agregam verdadeiro ganho terapêutico ao tratamento ${ }^{27,28}$.

Outra questão importante e não abordada pelos entrevistados é que as novidades na terapêutica frequentemente se associam a informação incompleta ou imperfeita, representando a prescrição de um medicamento cuja gama de efeitos é ainda desconhecida; quanto mais novo um medicamento, menores as chances de que as evidências de segurança estejam amplamente conhecidas e disponíveis na literatura ${ }^{29}$. Efeitos adversos raros, porém graves, podem não ser identificados nas pesquisas clínicas anteriores ao registro e, para que sejam detectados, pode ser necessária a utilização continuada do medicamento por grande número de pacientes. Existem exemplos recentes de multas impostas à indústria farmacêutica por veicular informações falsas ou incompletas de ensaios clínicos, com risco aos pacientes ${ }^{30}$. É esperado que a inclusão nas LME privilegie medicamentos com suficiente tempo de uso e com perfil de segurança e efetividade mais bem delineados, observáveis apenas após alguns anos na fase de pós-comercialização ${ }^{29}$.

\section{O Conceito de Medicamentos Essenciais: Percepção e Prescrição}

No cenário ambulatorial, os médicos prescrevem considerando, principalmente, a oferta de medicamentos na unidade e a condição financeira dos pacientes. E destacam sua percepção de que aquele que o paciente pode comprar nem sempre é o melhor:

\footnotetext{
"Às vezes, eu faço a medicação que eu tenho para fazer [...] eu sei que a população não vai ter condições de comprar a mais adequada.[...]". (Lucio)

"Sinceramente, eu procuro ver o que tem na Farmácia, [...]. Se não tiver na Farmácia, pergunto se ele pode comprar, se ele puder, eu passo o melhor. Senão eu passo um que vá mais ou menos". (Luisa)
}

A falta de medicamentos essenciais no SUS é uma realida$\mathrm{de}^{31} \mathrm{a}$ considerar no momento da prescrição. $\mathrm{O}$ alto percentual de prescrições de acordo com a LME visto em pesquisas anteriores pode estar relacionado não com o fato de o medicamento estar na lista, mas com o fato de estar disponível ${ }^{8,32}$. Os depoimentos acima indicam que esta preocupação se sobrepõe ao julgamento clínico-terapêutico. Por outro lado, considerar a condição econômica do paciente não é apenas lícita, mas ética. Ocorre que há uma série de parâmetros a seguir no caminho para orientar a escolha terapêutica, que envolvem a necessi- 
dade de saúde, a indicação e os fatores ligados ao acesso. Em contrapartida, parece haver nas falas uma associação entre o preço e a qualidade do que é prescrito, sendo o "melhor" visto como o mais caro.

A experiência individual do médico com o medicamento, também citado por Uchoa e Camargo ${ }^{26}$ como fator determinante da prescrição, apareceu tanto no discurso dos prescritores que não tiveram contato com uma LME, como no daqueles que tiveram, independentemente do nível de complexidade da unidade:

\section{"Tem muito a questão da medicação, a gente confiar na medi- cação. E já ter prática com aquela medicação. [...] a gente faz muita questão de experiência pessoal [...]" (Lucio Mauro) \\ “Eu uso medicação que eu tenho, é confiança, que eu já tenho experiência em usar. [...] Então, eu tenho os meus medica- mentos de uso". (Levi)}

É importante que os médicos conheçam os medicamentos que prescrevem; para tanto, devem formar um rol de medicamentos que utilizam frequentemente em sua prática clínica e para os quais tenham em mãos evidências atualizadas $^{33}$. Esta é a boa prática. Todavia, é preciso diferenciar esta posição daquela centrada na prática clínica individual, não aderente à Medicina Baseada em Evidência $(\mathrm{MBE})^{21}$. Neste sentido, a posição "eu tenho os meus medicamentos" pode ser contrária à farmacologia. Sem conhecer, a priori, as características dos pacientes, pautadas em condições físicas, psicológicas e sociais que podem demandar ações mais específicas e direcionadas, não é possível discriminar medicamentos com farto fundamento sobre sua atividade, indicação, eficácia e segurança ${ }^{34}$.

Um determinante preocupante para a prescrição de medicamentos surgiu nas falas, ainda que com menor intensidade: a solicitação do paciente:

"O que eu prescrevo bastante é o que a população mais procura. Aqui também procuram muita medicação de tarja preta". (Luisa)

Medicamentos assumem diferentes significados na sociedade moderna e exercem "funções" não "medicamentosas", mas terapêuticas, como amenizar dores sociais, por exemplo. Isto tem levado à procura e ao uso sem questionamento e à medicalização ${ }^{35}$. Ceder ao pedido do paciente não é prática legítima, tampouco ética. Há notoriamente entre o profissional de saúde e aquele que o procura uma grande assimetria de informação idônea ${ }^{35}$. Cabe ao prescritor que estuda e está atualizado - e, sobretudo, responsável e preocupado com a saúde do seu paciente - contrapor argumentos racionais às expectativas do paciente. Resultados de uma pesquisa realizada por Oliveira mostram que as mulheres conhecem que existe a possibilidade de dependência de benzodiazepínicos, mas não reconhecem os riscos envolvidos no uso desses medicamentos ${ }^{36}$.

\section{NOTAS FINAIS}

O estudo envolveu prescritores em cenários de baixa e média complexidade do SUS nas cinco regiões brasileiras. Os resultados das falas mostram baixa adesão às listas de medicamentos essenciais (LME), mesmo que maior adesão fosse esperada tendo em vista o tempo de atuação desses profissionais e os esforços na elaboração e divulgação das listas nacionais. Ainda que tenham tido algum contato prévio com uma LME, esta não é valorizada como fonte de informações para a prescrição baseada em evidências. Muitos prescritores entendem a LME como empecilho burocrático à prescrição, além de não verem nela utilidade prática. Não é de estranhar, portanto, o distanciamento dos prescritores em relação ao conceito de medicamentos essenciais, tendo em vista o papel da lista enquanto concretização deste conceito.

Houve cuidado em garantir a diversidade dos entrevistados, sua distribuição regional e em instâncias de gestão com e sem uma LME ativa. Houve também atenção para incluir entrevistados de diferentes cenários de prática. No entanto, destaca-se que o grupo de entrevistados não constitui uma amostra representativa - o estudo teve por objetivo compreender a percepção dos prescritores e, para tanto, se valeu de desenho da pesquisa qualitativa em saúde. Desta forma, os resultados não podem ser extrapolados para todos os médicos em atuação na baixa e média complexidade no SUS. Por outro lado, conferem uma gama de informações importantes sobre o grupo estudado e apontam práticas e possíveis dificuldades coletivas.

Não se conhece outro estudo de âmbito nacional, no País, com a abordagem adotada. Assim, foi empreendido o desenho metodológico da forma mais inclusiva possível. O ponto a salientar é a amplitude do conceito de "contato" adotado, motivado para ampliar ao máximo as oportunidades de aproximação entre os entrevistados e o objeto.

Espera-se que este estudo possa ajudar a superar as barreiras encontradas na adoção das LME nos cenários de prática do SUS. E também que possa embasar ações de educação continuada com os prescritores do SUS em relação ao conceito dos medicamentos essenciais e a uma prática de utilização das LME condizente com a boa prática baseada em evidências. 


\section{AGRADECIMENTOS}

Os autores agradecem o financiamento da Capes - Programa de Excelência Acadêmica Proex-Capes Discente - e aos pesquisadores de campo Flávia Alves Fernandes de Oliveira, Flávia Barroso Teixeira, Gilvan da Silva Araújo, Juliana Batista de Souza e Tatiana de Jesus Nascimento Ferreira.

\section{REFERÊNCIAS}

1. World Health Organization. Continuity and Change: Implementing the third WHO Medicines Strategy (20082013). Department of Essential Medicines and Pharmaceutical Policies. WHO; 2009. 37p.

2. World Health Organization. Drug and therapeutics committees: a practical guide Department of Essential Drugs and Medicines Policy. Managing the formulary process. Geneva: WHO; 2003.

3. Pepe VLE, Osorio-de-Castro CGS, Luiza VL. A Relação Nacional de Medicamentos Essenciais: um instrumento da Política Nacional de Medicamentos na garantia do acesso. In: Projeto Inovação em Saúde. Presidência Fiocruz (Org.). Inovação, pesquisa e acesso a medicamentos no Brasil. 1.ed. Rio de Janeiro: Fiocruz; 2008. p.319-334.

4. Brasil. Ministério da Saúde. Relação Nacional de Medicamentos Essenciais - RENAME 2010. Brasília (DF): Ministério da Saúde; 2010. (Série B. Textos Básicos de Saúde)

5. Brasil. Ministério da Saúde. Secretaria de Ciência, Tecnologia e Insumos Estratégicos. Departamento de Assistência Farmacêutica e Insumos Estratégicos. Formulário Terapêutico Nacional 2010. Rename 2010. Brasília: Ministério da Saúde; 2010. 1140 p. (Série B. Textos Básicos de Saúde).

6. Brasil. Ministério da Saúde. Organização Pan-Americana da Saúde. Avaliação da assistência farmacêutica no Brasil: estrutura, processo e resultados. Brasília: Organização Pan-Americana da Saúde; 2005.

7. Guerra Jr AA, Acúrcio FA, Gomes CAP, Miralles M, Girardi SN, et al. Disponibilidade de medicamentos essenciais em duas regiões de Minas Gerais, Brasil. Rev Panam Salud Publica 2004; 15(3).

8. Dal Pizzol TS, Trevisol DJ, Heineck I, Flores LM, Camargo AL, Köenig A et al .Adesão a listas de medicamentos essenciais em municípios de três estados brasileiros. Cad. Saúde Pública 2010; 26(4).

9. Vieira FS, Zucchi P. Demandas judiciais e assistência terapêutica no Sistema Único de Saúde. Rev. Assoc. Med. Bras. 2009; 55(6).

10. Cabana MD, Rand CS, Powe NR, Wu AW, Wilson MH, Abboud P, Rubin HR. Why don't physicians follow clinical practice guidelines? A framework for improvement. JAMA 1999; 20/282 (15)

11. Pepe VLE, Ventura M, Sant'ana JMB, Figueiredo TA, Souza VR, Simas L, et al. Caracterização de demandas judiciais de fornecimento de medicamentos "essenciais" no Estado do Rio de Janeiro, Brasil. Cad. Saúde Pública 2010; 26(3).

12. Soares JCRS, Depra AS. Ligações perigosas: indústria farmacêutica, associações de pacientes e as batalhas judiciais por acesso a medicamentos. Physis 2012; 22(1).

13. Magarinos-Torres R. A seleção de medicamentos essenciais na percepção de gestores e prescritores do Sistema Único de Saúde. Rio de Janeiro; 2011. Doutorado [Tese] - Fundação Oswaldo Cruz.

14. Bardin, L Análise de Conteúdo Edições Loyola, 5ª edição, 2010. 281 p.

15. Gomes R. A análise de dados em pesquisa qualitativa. In: Deslandes SF, Neto OC, Gomes R, Minayo MCS (organizadora). Pesquisa social: teoria, método e criatividade. Petrópolis, RJ: Vozes, 2010. 29ạ edição. Capítulo IV. Pags 79-108.

16. Brasil. Ministério da Saúde. Secretaria de Políticas de Saúde. Departamento de Atenção Básica. Política Nacional de Medicamentos. Série C Programas, projetos e Relatórios. Brasília: Ministério da Saúde; 2001. 40p.

17. Brasil. Ministério da Saúde. Secretaria de Ciência, Tecnologia e Insumos Estratégicos. Departamento de Ciência e Tecnologia. Série B. Textos Básicos em Saúde. Agenda nacional de prioridades de pesquisa em saúde. Brasília: Ministério da Saúde; 2008.

18. Oliveira MA, Bermudez JAZ, Osório-de-Castro CGS. Assistência Farmacêutica e Acesso a Medicamentos. Rio de Janeiro: Ed. Fiocruz; 2007.

19. Gustafsson LL, Wettermark B, Brian G, Karlsson EA, BergmanU, Hasselstrom J et al. The 'Wise List' - A Comprehensive Concept to Select, Communicate and Achieve Adherence to Recommendations of Essential Drugs in Ambulatory Care in Stockholm. Basic \& Clinical Pharmacology \& Toxicology 2011; 108:224-233.

20. Pepe VLE, Osorio-de-Castro CGS. A interação entre prescritores, dispensadores e pacientes: informação compartilhada como possível benefício terapêutico. Cad. Saúde Pública 2000;16(3).

21. Sackett DL, Rosenberg WMC, Gray JAM, Haynes RB, Richardson WS. Evidence based medicine: what it is and what it isn't. BMJ 1996; 312: 71

22. Brasil. Portaria № 533, de 28 de março de 2012. Estabelece o elenco de medicamentos e insumos da Relação Nacional de Medicamentos Essenciais (RENAME) no âmbito do Sistema Único de Saúde (SUS). 29 de março de 2012. 
23. Mintzberg H, Lampel J, Quinn JB, Ghoshal S. A organização profissional. In: O processo da estratégia: conceitos, contextos e casos selecionados. Artmed; 2003. pg 311-335.

24. Sharma S; Kh R; Chaudhury RR. Attitude and opinion towards essential medicine formulary. Indian J Pharmacol 2010; 42(3):150-2.

25. Sansgiry SS, Hayes JD, Rice GK. Attitudes of physicians toward formularies and services provided by pharmacists. Manag Care Interface. 2003;16(2):34-8.

26. Uchoa SAC e Camargo KR. Os protocolos e a decisão médica: medicina baseada em vivências e ou evidências?. Ciênc. Saúde Coletiva 2010;15(4).

27. Angel M. A verdade sobre os laboratórios farmacêuticos. Rio de Janeiro: Record; 2007.

28. Comparative advantages of new drugs: French authorities are not sufficiently demanded. Prescrire International 2005; 14(77):75-79

29. Wannmacher, L. Medicamentos essenciais: vantagens de trabalhar com este contexto. Uso Racional de Medicamentos: Temas Selecionados 2006; 3(2):1-6.

30. Whalen J, Barrett D, Loftus P. Glaxo in \$3 Billion Settlement. Disponível em: http:/ / online.wsj.com/article/SB10 001424052702304299704577502642401041730.html

31. Souza JM, Vinholes ER, Galato SCTD. Avaliação dos indicadores de prescrição e da demanda atendida de medicamentos no Sistema Único de Saúde de um município do Sul do Estado de Santa Catarina. Rev Ciênc Farm Básica Apl., 2012;33(1):107-113

32. Santos V dos e Nitrini SMOO. Indicadores do uso de medicamentos prescritos e de assistência ao paciente de serviços de saúde. Rev. Saúde Pública 2004; 38(6):819-834.
33. Organização Mundial da Saúde (OMS). Departamento de Medicamentos Essenciais e Política de Medicamentos. Guia do Instrutor em Práticas da Boa Prescrição Médica; 2001.

34. Nobre MRC, Bernardo WN, Jatene FB. A prática clínica baseada em evidências. Parte I: questões clínicas bem construídas. Rev. Assoc. Med. Bras. 2003; 49(4).

35. Guedes CR, Nogueira MI Camargo Jr., Kenneth R. de. Os sofredores de sintomas indefinidos: um desafio para a atenção médica?.Physis 2009;19(3).

36. Oliveira EM. Saúde Mental e mulheres: sobrevivência, sofrimento e dependência química lícita. Edições UVA; 2000. $151 p$.

\section{CONTRIBUIÇÃO DOS AUTORES}

Rachel Magarinos-Torres foi responsável pela coleta e análise dos dados, discussão e redação do artigo. Claudia Garcia Serpa Osorio-de-Castro e Vera Lúcia Edais Pepe orientaram a tese que deu origem ao artigo e trabalharam na discussão e redação do texto. Ângela Escher e Rosângela Caetano colaboraram na discussão dos resultados e revisão do texto.

\section{CONFLITO DE INTERESSES}

Declarou não haver.

\section{ENDEREÇO PARA CORRESPONDÊNCIA}

Rachel Magarinos Torres

Faculdade de Farmácia - Universidade Federal Fluminense

Rua Mário Viana, 523

Santa Rosa - Niterói

CEP 24241-000 - RJ

E-mail: rmtorres@id.uff.br; racheltorres.uff@gmail.com 